\title{
Experimental Analysis of Single and Multiple Antenna Units in Uplink of Radio-over-Fiber Distributed Antenna System
}

\author{
Anthony E. Aighobahi, Philippos Assimakopoulos, and Nathan J. Gomes \\ Communications Research Group, University of Kent, Canterbury, UK \\ Email: aea25@kent.ac.uk
}

\begin{abstract}
Increasing the number of antennas either at the transmitter or receiver has been shown to improve system reliability without occupying additional spectrum. In this paper, we experimentally investigate the error vector magnitude (EVM) of single and multiple remote antenna units (RAU) focusing on uplink transmission. We demonstrate that for 64-QAM modulation, the EVM requirement of $6.5 \%$ could be achieved with multiple separated RAUs in situations where a single RAU fails to meet this requirement. The EVM result was obtained as the transmitting device was placed at different locations in a typical office environment with OFDM signals gathered through the RAUs and brought back to a central unit for processing. The EVM results show that using multiple RAUs and an efficient signal combining technique, here, maximal ratio combining (MRC), the EVM performance could reduce by approximately $2 \%$ when the distance between the RAUs was $0.3 \mathrm{~m}$ and further reduced by $4 \%$ and $6 \%$ when the inter-RAU distance was $2 \mathrm{~m}$ and $4 \mathrm{~m}$, respectively, compared to a single RAU.
\end{abstract}

Keywords- Distributed antenna system; Radio-over-fiber; Maximal ratio combining; Spatial diversity

\section{INTRODUCTION}

As the demand for higher data rates by growing numbers of wireless users increases, it has become necessary to develop a system that will improve wireless coverage and capacity, especially in an indoor environment where $80-90 \%$ of wireless data traffic originates[1]. One approach is to redesign the network infrastructure, and the most popular methods currently deployed are femtocells, relays and distributed antenna systems (DAS). DAS has attracted much attention [24] because of its ability to produce high quality coverage, low interference and high signal-to-noise ratio (SNR) and, using different techniques such as higher order modulation, orthogonal frequency-division multiplexing (OFDM), advanced coding schemes and, more recently, multiple-input multiple-output (MIMO) schemes, it can widely improve wireless access rates.

In DAS, remote antenna units (RAUs) are geographically deployed and connected to a central unit (CU) using either an out band radio link, dedicated cables, digital optical network or Radio-over-fiber (RoF) link. A RoF link has key advantages such as simple configuration, low loss to improve signal transmission and low infrastructure cost. The RAUs are merely a distributed set of antennas, with little or no processing capability, that exchange radio frequency (RF) signals with the $\mathrm{CU}$ where all the signal processing such as modulation, demodulation, multiplexing, de-multiplexing, handover, diversity and protocol transformations are carried out[5].

MIMO has been adopted in most new wireless communication standards, such as WiFi, LTE and WiMAX, to provide increased capacity and spectral efficiency while improving the link reliability compared to the traditional single antenna technology. It helps to solve the fading problem experienced by conventional wireless systems where the received signal quality varies unpredictably causing the signal strength to reduce. The integration of RoF-DAS and MIMO can bring about improvement in throughput performance.

In [6] we investigated the throughput performance of a commercial MIMO access point in a DAS where we demonstrated the benefits of transmitting MIMO signals using RoF-DAS since it enables the separation of MIMO antennas to allow an additional degree of freedom. The usual small antenna separation limits the system capacity due to the relatively high correlation of the signals being received. Another paper also investigated $3 \times 3$ MIMO access point to show improved throughput performance when using RoFDAS [7]. These works were based on commercial products and the specific MIMO algorithms used were not known.

The spatial diversity technique is a form of MIMO where multiple transmitting or receiving antennas enable a variety of signal paths. The advantage of spatial diversity is that the chances of correctly receiving the data are increased. Maximal ratio combining (MRC) is a spatial diversity technique that combines the signals from multiple diversity antennas, amplifying the better signal while the poorer signal is attenuated to achieve the maximum spatial diversity order when there is less spatial correlation between the antennas.

In this paper, we investigate the potential benefits of the Maximal Ratio Combining (MRC) algorithm in RoF-DAS uplink. This paper compares the results of the performance using single and multiple RAUs in uplink transmission. The experimental setup is described in Section II whist the results 


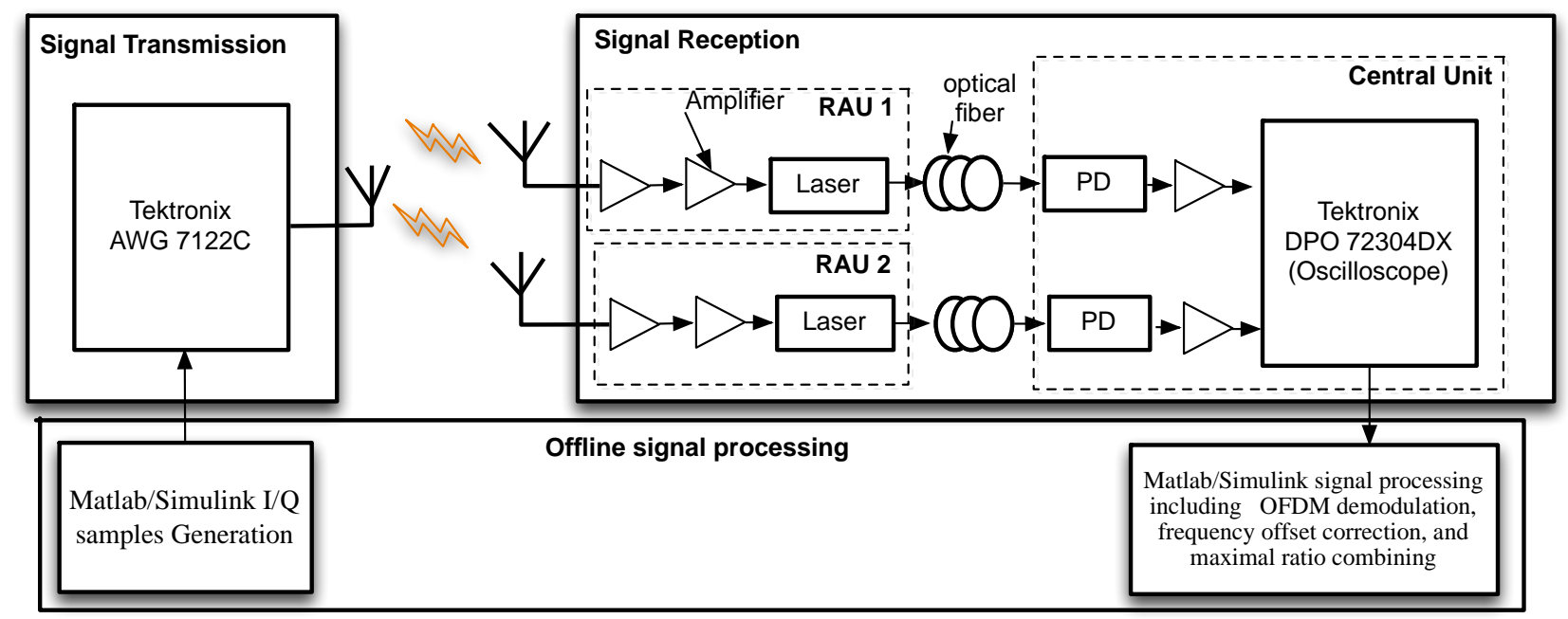

Fig1. Experimental Set-up of the Maximal Ratio combining (MRC) in uplink signal transmission of RoF-DAS.

analysis is shown in Section III. Conclusion are presented in Section IV

\section{EXPERIMENTAL SETUP}

Fig. 1 shows the experimental setup of the uplink signal transmission of RoF-DAS. The transmitted signal was processed using MATLAB/Simulink software. Pseudorandom data were generated and converted to parallel sets of bits for the 64-QAM-OFDM processing. The OFDM signal processing includes 2048 IFFT size, 10 pilot subcarriers, 92 zeros and CP size of 1/4 (other parameters are given in Table 1). The I/Q samples were downloaded onto a Tektronix Arbitrary Waveform generator (AWG) 7122C and unconverted to a Radio Frequency (RF) signal at a carrier frequency of $2.4 \mathrm{GHz}$, and the RF signal was transmitted to the RAUs. The receiver (Tektronix DPO 72304DX) was placed in the central unit while the transmitter and RAU were in a separate room. The RAUs receive the transmitted signal and perform RF amplification and electro-optic conversion with a directly modulated laser diode. The gains of the amplifiers were set to use the linear region of the laser diode and at the point where the received signal would experience less noise and distortion. Both laser diodes were connected to 25-meter lengths of single mode fiber (SMF) and then to the photodiodes in the CU. The RoF link, consisting of the direct modulated laser, optical fiber and direct detection Photodiode (PD) had a loss of $25 \mathrm{~dB}$; therefore we place an amplifier with $20 \mathrm{~dB}$ gain before the oscilloscope to increase the level of the received signal. The received signals are saved in MATLAB format in the oscilloscope so that they can be demodulated offline and combined using the MRC algorithm.

Fig 2 shows the layout of the testing room where the RAUs and the transmitter were placed. One of the benefits of DAS is it enables antenna separation and larger distance can reduce spatial correlation to provide improved signal reception possibilities for the wireless user. Measurements were thus carried out when the two RAUs were separated at different distances to examine this effect. At first, the RAUs were placed relatively close to each other (Ta1 and Ta2) at a separation of $0.3 \mathrm{~m}$; thereafter, the RAUs were separated (Tb1 and $\mathrm{Tb} 2$ ) at a distance of $2 \mathrm{~m}$, and then further separated (Tc1 and $\mathrm{Tc} 2$ ) at a distance of $4 \mathrm{~m}$. The testing room is a typical office room with common office furniture (desks, chairs and computers). The RAUs were placed on a wooden platform extension on some of the desks so that the signals were generally radiating above the other desks. The Error Vector Magnitude (EVM) measurement was obtained after positioning the transmitting AWG (the mobile user) at different locations A, B, C (1-4) in the room, to determine the signal transmission quality.

TABLE I. OFDM SIGNAL PARAMETERS.

\begin{tabular}{|c|c|c|}
\hline \multicolumn{2}{|c|}{ Parameters } & Values \\
\hline \multicolumn{2}{|c|}{ Carrier Frequency } & $2.4 \mathrm{GHz}$ \\
\hline \multirow{3}{*}{ OFDM } & IFFT Length & 2048 \\
\cline { 2 - 3 } & Number of zeros & 384 \\
\cline { 2 - 3 } & Number of Pilots & 8 \\
\cline { 2 - 3 } CP size & $1 / 4$ \\
\hline \multicolumn{2}{|c|}{ QAM Modulation } & 64 \\
\hline \multicolumn{2}{|c|}{ Bandwidth } & $32 \mathrm{MHz}$ \\
\hline \multicolumn{2}{|c|}{ RAU Transmit power } & $7 \mathrm{dBm}$ \\
\hline \multicolumn{2}{|c|}{ RoF link gain } & $-25 \mathrm{~dB}$ \\
\hline
\end{tabular}




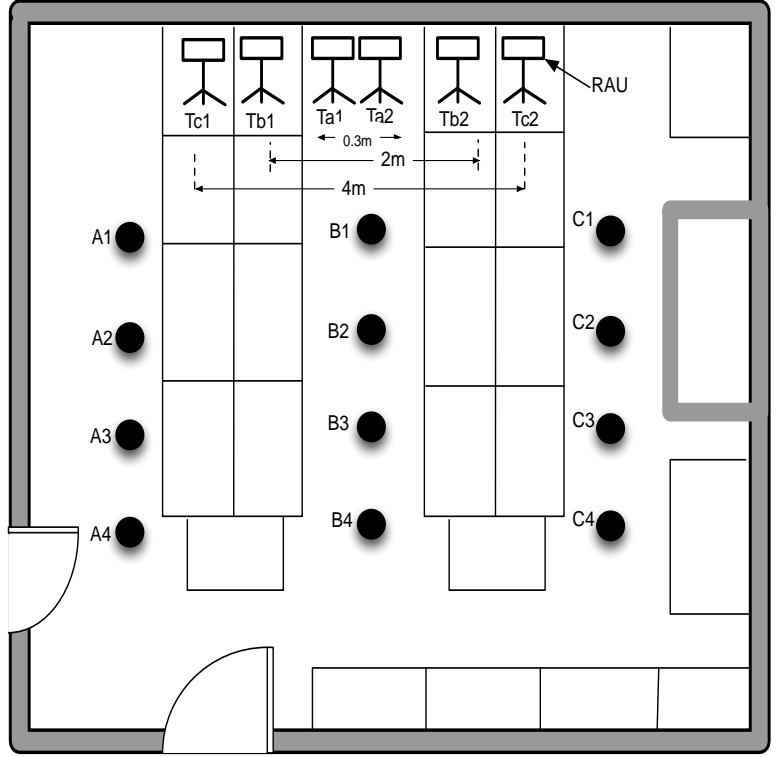

Fig. 2 Experimental layout of an open plan office. A, B, C (1-4) represent the positions of the mobile user (the AWG).

To evaluate the performance advantage of the two receiver antenna system in RoF-DAS, we also obtained EVM results for the single antenna transmission to be used as a baseline for comparison. The EVM measurement for this case was taken when the RAU was at location Ta1 shown in Fig. 2.

\section{EXPERIMENTAL RESULTS}

Figures 3, 4 and 5 show the EVM results obtained from the 12 transmitting locations (four in each row) in the typical office environment for the single RAU case and for the two RAU case with the different inter-RAU separations. The graphs also show the standard deviation results at each measurement point. The total transmitter power was set to 7 $\mathrm{dBm}$ and the gain of the amplifier in the RAU was set to 20 $\mathrm{dB}$ to ensure that the laser diode was driven in its linear region. The RoF link has a loss of $25 \mathrm{~dB}$, therefore an amplifier was placed before the oscilloscope with $20 \mathrm{~dB}$ gain to reduce the loss of the link and to allow correct demodulation of the received signal. The RAU collects the signal from each location in the room as the transmitter (AWG) is moved around, after which it is transported back to the CU, and the EVM of the signal captured on the oscilloscope was measured using MATLAB offline processing. The EVM gives an indication of transmission quality and a minimum value is often specified in advanced radio systems with complex modulation schemes. EVM and Carrier-to-noise ratio (CNR) are related through the following equation [8]:

$$
C N R=-(r+20 \log [E V M /(100 \%)])
$$

where $r$ is the peak-to-average energy ratio of the QAM constellation and is $3.7 \mathrm{~dB}$ for 64QAM and $4.2 \mathrm{~dB}$ for 256QAM. Using this equation, the EVM requirement becomes $6.5 \%$ for 64-QAM and 3.1\% for 256-QAM [8].
The threshold for EVM for 64QAM modulation is $6.5 \%$ shown by the blue line in Figs. 3, 4 and 5, the lower EVM result would thus enable higher achievable data rate. The results obtained from $2 \mathrm{~m}$ and $4 \mathrm{~m}$ RAU separation distance when the two RAUs received the signal are below the threshold EVM for 64-QAM modulation and therefore would enable these higher data rates. This was made possible by DAS since it allows antenna separation and thus the RAUs receives signal through a variety of paths. Furthermore, the results clearly show that with two RAUs together and the MRC signal processing technique, the EVM could reduce by a $2 \%$ when the inter-RAU separation distance is $0.3 \mathrm{~m}$, and $4 \%$ at $2 \mathrm{~m}$ separation distance and $6 \%$ at $4 \mathrm{~m}$ as shown in Table 2.

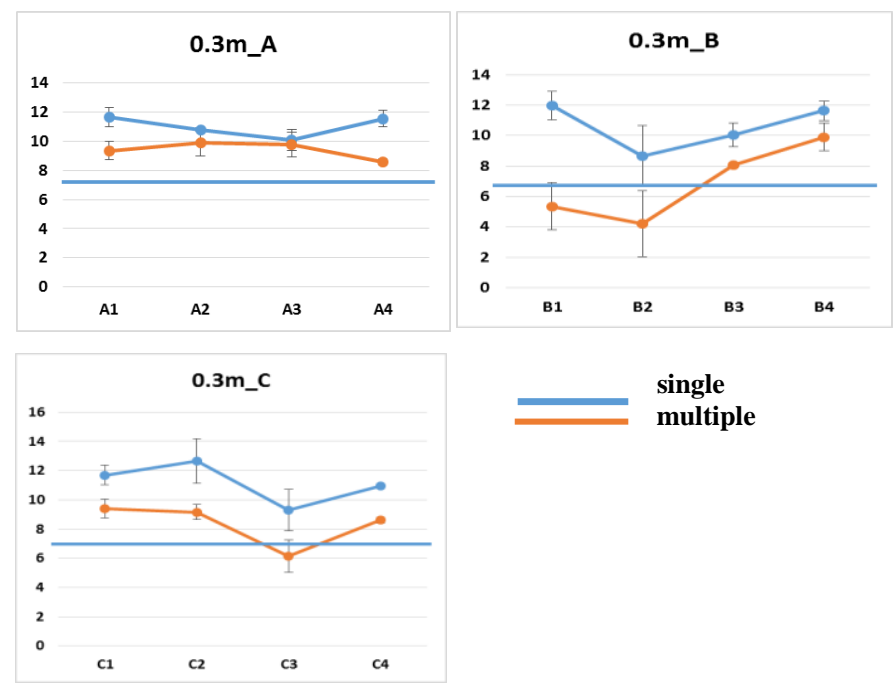

Fig 3. EVM result of rows A, B and C for single and multiple unit at inter-RAU separation distance of $0.3 \mathrm{~m}$.

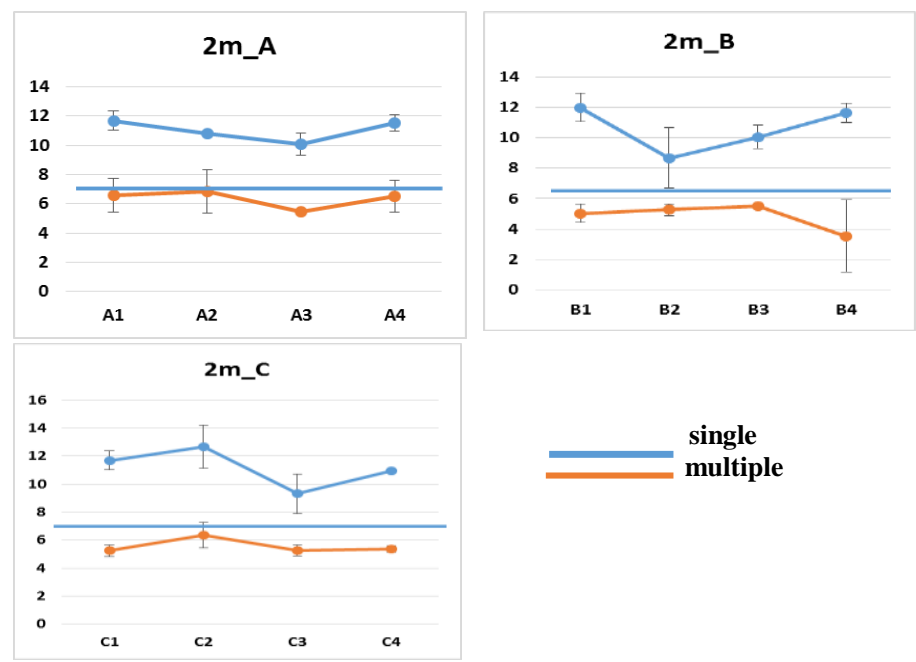

Fig 4. EVM result of rows A, B and C for single and multiple unit at inter-RAU separation distance of $2 \mathrm{~m}$. 

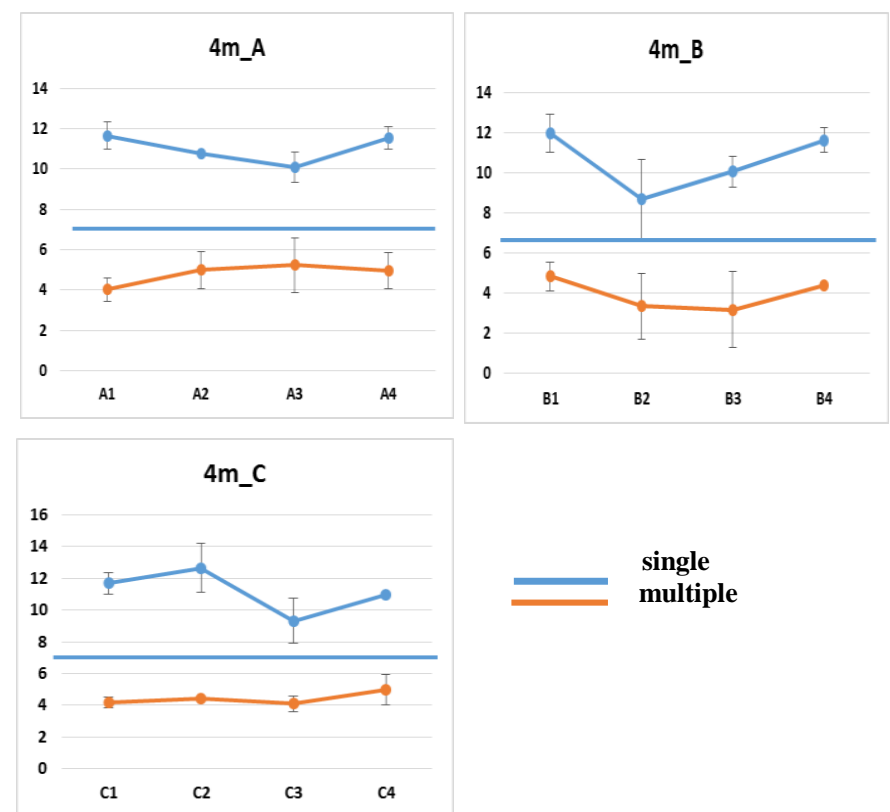

Fig 5. EVM result of rows A, B and C for single and multiple unit at inter-RAU separation distance of $4 \mathrm{~m}$

TABLE II. REDUCTION IN THE MEAN EVM PER ROW COMPARED TO THE SINGLE ANTENNA CASE AND STANDARD DEVIATION RESULTS.

\begin{tabular}{|c|r|r|r|r|r|r|}
\hline & \multicolumn{2}{|c|}{$0.3 \mathrm{~m}$} & \multicolumn{2}{c|}{$2 \mathrm{~m}$} & \multicolumn{2}{c|}{$4 \mathrm{~m}$} \\
\hline & \multicolumn{1}{|c|}{$\mathrm{R}$} & \multicolumn{1}{c|}{$\mathrm{SD}$} & \multicolumn{1}{c|}{$\mathrm{R}$} & \multicolumn{1}{c|}{$\mathrm{SD}$} & \multicolumn{1}{c|}{$\mathrm{R}$} & \multicolumn{1}{c|}{$\mathrm{SD}$} \\
\hline $\mathrm{A}$ & 1.62 & -0.56 & 4.66 & -0.81 & 6.21 & -0.57 \\
\hline $\mathrm{B}$ & 2.70 & 0.05 & 5.13 & -0.29 & 6.08 & -0.82 \\
\hline $\mathrm{C}$ & 3.60 & 0.36 & 5.00 & -0.30 & 5.96 & -0.65 \\
\hline
\end{tabular}

\section{CONCLUSION}

We demonstrated that the larger antenna separation distances made possible by RoF-DAS makes it possible to achieve higher spatial diversity since there is less correlation between the receiving antennas. We presented the EVM results of the performance of uplink transmission of single and multiple remote antenna units and we observed that when the RAUs receives the signal from two receiving antennas, there was improved EVM performance of approximately $2 \%$ with antenna separation distance of $0.3 \mathrm{~m}$ and $4 \%$ and $6 \%$ with antenna separation distance of $2 \mathrm{~m}$ and $4 \mathrm{~m}$, respectively, using the MRC signal processing combing technique. The EVM performance of the separated antenna distance of $2 \mathrm{~m}$ and $4 \mathrm{~m}$ are below the EVM required for 64-QAM modulation.

\section{ACKNOWLEDGMENT}

The authors are grateful to Dr. Anthony Nkansah for advice on the measurements.

\section{REFERENCES}

[1] Z. F. Fengming Cao, "Joint proportional fair user scheduling for in- building distributed antenna systems " IET Commun., vol. 7 pp. 1802-1809 2013.

[2] T. Alade, H. Zhu, and H. Osman, "The impact of antenna selection and location on the performance of DAS in a multi-storey building," IEEE Wireless Communications and Networking Conference (WCNC), pp. 3213 - 3218, 2013

[3] H. Zhu, "Performance Comparison Between Distributed Antenna and Microcellular Systems," IEEE Journal on Selected Areas in Communications, pp. 1151 - 1163, 2011.

[4] H. Osman, H. Zhu, and T. Alade, "Distributed antenna systems with frequency reuse," Persona IEEE 22nd International Symposium on Indoor and Mobile Radio Communications (PIMRC), pp. 1855 - 1859, 2011.

[5] A. Attar, H. Li, and V. C. M. Leung, "Applications of Fiberconnected Distributed Antenna Systems in Broadband Wireless Access," International Conference on Computing, Networking and Communications (ICNC), pp. 623 - 627, 2012.

[6] Y. Fan, A. E. Aighobahi, N. J. Gomes, K. Xu, and J. Li, "Performance analysis of commercial multiple-input-multipleoutput access point in distributed antenna system," Optical Express, vol. 23, pp. 500-7513 2015.

G. S. Gordon, M. J. Crisp, R. V. Penty, and I. H. White, "Experimental Evaluation of Layout Designs for $3 \times 3$ MIMOEnabled Radio-Over-Fiber Distributed Antenna Systems," IEEE Transactions on Vehicular Technology, pp. 643 - 653, 2014. W. Music. (2001). TG3 measurement accuracy, IEEE P802.15 working group for wireless personal area networks. Available: http://www.ieee802.org 